\title{
Signatures of the Tricritical Point in QCD
}

\author{
M. Stephanov ${ }^{1}$, K. Rajagopal ${ }^{2}$ and E. Shuryak ${ }^{3}$ \\ ${ }^{1}$ Institute for Theoretical Physics, State University of New York, Stony Brook, NY 11794-3840 \\ ${ }^{2}$ Center for Theoretical Physics, Massachusetts Institute of Technology, Cambridge, MA 02139 \\ 3 Department of Physics and Astronomy, State University of New York, Stony Brook, NY 11794-3800
}

(June 1, 1998; ITP-SB-98-39, MIT-CTP-2748, SUNY-NTG-98-17)

\begin{abstract}
Several approaches to QCD with two massless quarks at finite temperature $T$ and baryon chemical potential $\mu$ suggest the existence of a tricritical point on the boundary of the phase with spontaneously broken chiral symmetry. In QCD with massive quarks there is then a critical point at the end of a first order transition line. We discuss possible experimental signatures of this point, which provide information about its location and properties. We propose a combination of event-by-event observables, including suppressed fluctuations in $T$ and $\mu$ and, simultaneously, enhanced fluctuations in the multiplicity of soft pions.
\end{abstract}

In QCD with two massless quarks, a spontaneously broken chiral symmetry is restored at finite temperature. It can be argued [1,2] that this phase transition is likely second order and belongs to the universality class of $O(4)$ spin models in 3 dimensions. If this transition is indeed second order, QCD with two quarks of nonzero mass has only a smooth crossover as a function of $T$. Although not yet firmly established, this picture is consistent with present lattice simulations and many models.

At zero $T$ several models suggest [3] 7] that the chiral symmetry restoration transition at finite $\mu$ is first order. Assuming that this is the case in QCD, one can easily argue that there is a tricritical point in the $T \mu$ phase diagram, where the transition changes from first to second order. The nature of this point can be understood by considering the Landau-Ginzburg effective potential for the order parameter of chiral symmetry breaking, $\phi=(\sigma, \boldsymbol{\pi}) \sim\langle\bar{\psi} \psi\rangle:$

$$
\Omega(\phi)=a \phi^{2}+b\left(\phi^{2}\right)^{2}+c\left(\phi^{2}\right)^{3} .
$$

The coefficients $a, b$, and $c>0$ are functions of $\mu$ and $T$. The second order phase transition line described by $a=0$ at $b>0$ becomes first order when $b$ changes sign. The critical properties of this point can be inferred from universality [6,7], and the exponents are as in the mean field theory (1).

In real QCD with nonzero quark masses the second order phase transition becomes a crossover and the tricritical point becomes a critical (second order) end-point of a first order phase transition line. Universality arguments [8,7] also predict that the end-point E in QCD with small quark masses is shifted with respect to the tricritical point $\mathrm{P}$ towards larger $\mu$ as shown in Fig. 1. It can also be argued [6,7] that the point $\mathrm{E}$ is in the universality class of the Ising model in 3 dimensions, because the $\sigma$ is the only field which becomes massless at this point. (The pions remain massive because of the explicit chiral symmetry breaking by quark masses.) In this paper we discuss experimental signatures of this critical end-point.

The position of the points $\mathrm{P}$ and $\mathrm{E}$ in two-flavor QCD was estimated recently using two different models (a

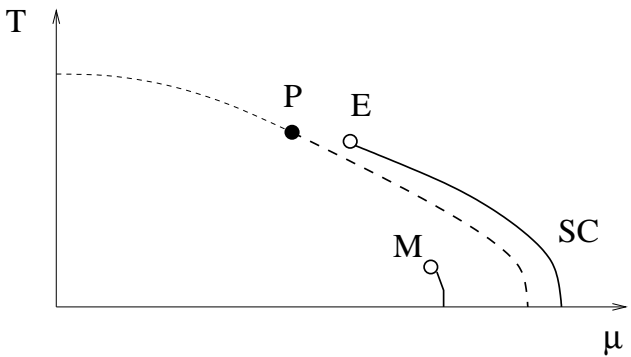

FIG. 1. The schematic phase diagram of QCD. The dashed lines represent the boundary of the phase with spontaneously broken chiral symmetry in QCD with 2 massless quarks. The point $\mathrm{P}$ is tricritical. The solid line with critical end-point $\mathrm{E}$ is the line of first order transitions in QCD with 2 quarks of small mass. The point $\mathrm{M}$ is the end-point of the nuclear liquid-gas transition probed in multifragmentation experiments. The superconducting phase of QCD [9,5, 6], marked SC, is not relevant to our discussion.

Nambu-Jona-Lasinio model respecting the global symmetries of QCD [6] and a random matrix model [7]) as $T_{P} \sim 100 \mathrm{MeV}$ and $\mu_{P} \sim 600-700 \mathrm{MeV}$. These are only crude estimates, since they are based on modeling the dynamics of chiral symmetry breaking only.

The third (strange) quark has an important effect on the position of the point $\mathrm{P}$ and, therefore, of the point $\mathrm{E}$. At $\mu=0$, if the strange quark mass $m_{s}$ is less than some critical value $m_{s 3}$, the second order finite $T$ transition becomes first order. This leads to a tricritical point in the $T m_{s}$ plane 10,11,21. Theoretically, the origin of this point is similar to the one we are discussing. In terms of eq. (11) the effect of decreasing $m_{s}$ is similar to the effect of increasing $\mu$ : the coefficient $b$ becomes negative. What is important is that, unlike $m_{s}, \mu$ is a parameter which can be experimentally varied.

Clearly, the physics of the $T \mu$ plane is as in Fig. 11 only for $m_{s}>m_{s 3}$. For $m_{s}<m_{s 3}$, the transition is first order already at $\mu=0$, and, presumably, remains first order at all nonzero $\mu$ [12]. As $m_{s}$ is reduced from infinity, the tricritical point $\mathrm{P}$ of Fig. 1 moves to lower $\mu$ until, at $m_{s}=m_{s 3}$, it reaches the $T$-axis and can be identified with the tricritical point in the $T m_{s}$ plane. The 
two tricritical points are continuously connected. We assume that $m_{s}>m_{s 3}$ which is consistent with the lattice studies of ref. 13]. What is important for us is that the qualitative effect of the strange quark is to reduce the value of $\mu_{P}$, and thus of $\mu_{E}$, compared to that in twoflavor QCD, since $\mu_{P}=0$ at $m_{s}=m_{s 3}$. This shift may be significant, since lattice studies show that the physical value of $m_{s}$ is of the order of $m_{s 3}$.

Analysis of particle abundance ratios in central heavy ion collisions 14 indicates that chemical freeze-out happens near the phase boundary, at a chemical potential $\mu \sim 500-600 \mathrm{MeV}$ at the AGS $(11 \mathrm{GeV} \cdot A)$, while at the SPS $(160-200 \mathrm{GeV} \cdot A)$ it occurs at a significantly lower $\mu \lesssim 200 \mathrm{MeV}$. In view of the effect of the strange quark just discussed, the estimated position of $\mathrm{P}$ and $\mathrm{E}$ [6,7] should be shifted from $\mu_{E} \sim 600-700 \mathrm{MeV}$ to lower $\mu$. Thus, it may well be between the SPS and the AGS values of $\mu$, and therefore the point $\mathrm{E}$ may be accessible at lower energy or non-central collisions at the SPS.

The strategy for finding the point $\mathrm{E}$ which we propose is based on the fact that this is a genuine critical point. Such a point is characterized by enhanced long wavelength fluctuations which lead to singularities in all thermodynamic observables. In the liquid-gas phase transition in water, critical opalescence signals the universal physics unique to the vicinity of the critical point. The signatures we propose can play an analogous role in QCD.

It is important to have control parameters which can be adjusted to vary the $\mu$ at which the system crosses the transition region, as shown in Fig. 2. For example, increasing the energy of the collision decreases this $\mu$. A somewhat similar effect may be achieved by increasing centrality. A third possibility would be to slice each event in rapidity, since $\mu$ will be greater at higher rapidity. This strategy could be useful at RHIC, if $\mathrm{E}$ were to lie at lower $\mu$ than is accessible at the SPS. We will call the control parameter which is varied " $x$ ", and take increasing $x$ to mean increasing collision energy or centrality or decreasing rapidity. Scanning in centrality will almost certainly be the easiest, since in any given run events with all impact parameters are present. However, scanning in energy yields a large variation in the $\mu$ at which the transition is crossed, whereas scanning in centrality may only provide fine tuning.

In this work we do not discuss initial equilibration and we choose to define the initial point, $\mathrm{I}(x)$, as the point at which compression has ended, most of the entropy is already produced, and approximately adiabatic expansion begins. The system will then follow some trajectory in the $T \mu$ plane characterized by the ratio of the baryon charge density to the entropy density, $n / s$, which is (approximately) conserved. Three trajectories are shown schematically in Fig. 2. (For realistic hydrodynamical calculations and discussion see, e.g., refs. 15, 16]).

Recall that the first order line in the $T \mu$ plane is actually a whole region of mixed phase, with the hidden pa-

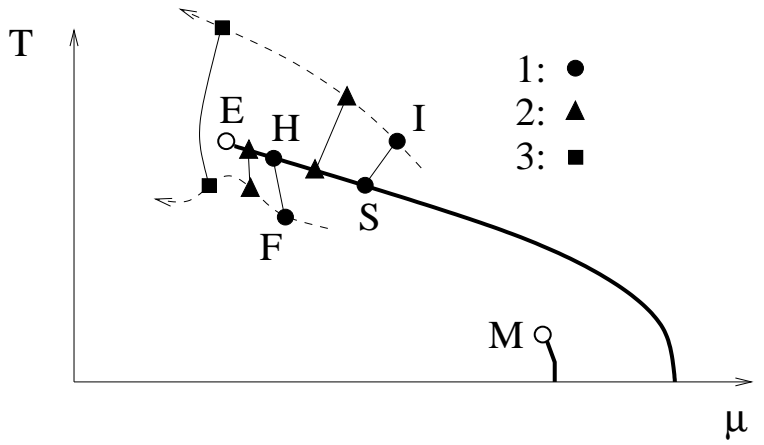

FIG. 2. Schematic examples of three possible trajectories for three values of $x$ on the phase diagram of QCD (see. Fig. 11. The points I, S, H and F on different trajectories are marked with different symbols. The dashed lines show the locations of the initial, I, and final, $\mathrm{F}$, points as $x$ is increased in the direction shown by the arrows.

rameter being the volume fraction of the two coexisting phases. The zig-zag shape occurs because the trajectory exits the mixed phase region at a point with the same value of the conserved $n / s$ that it had upon entering. Because $n / s$ is discontinuous at the first order line, this requires increasing $T$ and decreasing $\mu$ as latent heat is released 15, 16]. In Fig. 2, we use the following notation: $\mathrm{S}(x)$ for the "softest" point, $\mathrm{H}(x)$ for the "hottest" point and $\mathrm{F}(x)$ for the final thermal freeze-out after which no scattering occurs. (Note that at small values of $x$, at which the transition is first order, the trajectories are, in fact, likely to begin within the mixed phase region. The special case when $\mathrm{I}(x)$ coincides with $\mathrm{S}(x)$ leads to a local maximum of the QGP lifetime 17, 18 , which may be important for $J / \psi$ suppression [19].) Increasing $x$ will yield trajectories shifted to the left in Fig. 2, traversing the transition region at lower $\mu$ and higher $T$.

The existence of the end-point singularity, E, leads to the phenomenon which we refer to as the "focusing" of trajectories towards $\mathrm{E}$. The initial point $\mathrm{I}(x)$ and the beginning of the zig-zag $\mathrm{S}(x)$ depend on the control parameter $x$ more strongly than the zig-zag end-point $\mathrm{H}(x)$. The reason for this is that the point $\mathrm{H}(x)$ is always closer to E than $\mathrm{S}(x)$ (see Fig. 2). This focusing effect implies that exploring physics in the vicinity of the end-point singularity may not require a fine-tuned $x$. This situation resembles that in low energy nuclear collisions, in which the first order liquid-gas phase transition also has a critical end-point at a temperature of order $10 \mathrm{MeV}$ 20,21 (point $\mathrm{M}$ on Figs. 11 and 2). In such experiments, one varies control parameters to maximize the probability of multi-fragmentation. It was noticed long ago [20] how surprisingly easy it is to hit the critical region from a wide range of initial parameters.

Another aspect of the "focusing" arises via the divergence of susceptibilities, such as the specific heat capacity $c_{V}=T \partial s / \partial T$, at the endpoint E. As a result, the trajectories which pass near the critical point will linger 
there longer. This makes it likely that final freeze-out occurs at a temperature quite close to $T_{E}$, rather than below it. So, while scanning in some control parameter $x$ and measuring the positions of the points $\mathrm{F}(x)$, we may expect to find a bump in the vicinity of the point E. (See the lower dashed curve on Fig. 2.) At this point it is instructive to consider the dependence on another control parameter, the atomic weight $A$ of the colliding nuclei. If $A$ were infinite, the point $\mathrm{F}$ would be close to $T_{F}=0, \mu_{F}=m_{N}$. Thus, for $A$ large enough, the dotted curve in Fig. 2 moves down and the bump, and also all the other signatures described below, fade away. Experimentally, the $A$ dependence of the point $F$ has been established recently by the analysis of flow [16], Coulomb effects 22 and pion interferometry 23]. For example, in central S+S collisions at SPS $T_{F} \approx 140-150 \mathrm{MeV}$, while for $\mathrm{Pb}+\mathrm{Pb}$ it is only $T_{F} \approx 120 \mathrm{MeV}$.

We shall now discuss the signatures which directly reflect thermodynamic properties of the system near its critical point and are not very sensitive to the details of the evolution. With the advent of wide-solid-angle detectors like NA49 at CERN, it is now possible to make event-by-event measurements of observables which are proxies for the freeze-out $T$ and $\mu$ 24]. We argue that the event-by-event fluctuations in both quantities should be anomalously small for values of $x$ such that the system passes near the critical point. As has been suggested 25], event-by-event fluctuations of $T$ can be related by basic thermodynamics to the heat capacity at freeze-out

$$
\frac{(\Delta T)^{2}}{T^{2}}=\frac{1}{C_{V}} \text {. }
$$

The quantity $C_{V}$ is extensive, so $\Delta T \sim 1 / \sqrt{N}$ as expected, where $N$ is the number of particles in the system. If the specific heat $c_{V}$ diverges, the coefficient of $1 / \sqrt{N}$ vanishes and fluctuations of $T$ are suppressed. For freezeout in the crossover region, or in the hadronic phase just below the first order transition, $c_{V}$ is finite. (If freeze-out were to occur from the mixed phase, some linear combination of the two susceptibilities would be relevant.) As the critical point is approached from either the left or the right, $c_{V}$ diverges and $\Delta T \sqrt{N}$ decreases. Other susceptibilities, in particular, $-\partial^{2} \Omega / \partial \mu^{2}$, are also divergent. This implies that fluctuations of $\mu$ are also suppressed at the critical point. Experimentally, $\Delta T$ can be found via event-by-event analysis of $p_{T}$ spectra [25]. Fluctuations in $\mu$ correspond to event-by-event fluctuations in the baryon-number-to-pion ratio. The fluctuations in any experimental observable will receive contributions in addition to the thermodynamic ones we describe, for example from fluctuations in the flow velocity. We therefore expect that as the collision energy is increased so that the freeze-out point moves from right to left past the critical point, we will find minima (but not zeroes) in the widths of the distributions of those event-by-event observables which are well-correlated with $T$ and $\mu$.
Using universality, we can predict the exponents for the divergent susceptibilities at the point E. Very naively, one might think that the exponent describing the divergence of $C_{V}$ is $\alpha$, which is small for the 3-dimensional Ising model universality class: $\alpha \approx 0.12$. In fact the exponent for $C_{V}$ is significantly larger. This and the exponent for the $\mu$-susceptibility are determined by finding two directions, temperature-like and magnetic-field-like, in the $T \mu$ plane near point $\mathrm{E}$, following the standard procedure for mapping a liquid-gas transition onto the Ising model [26]. The two linear combinations of $T-T_{E}$ and $\mu-\mu_{E}$ corresponding to these directions should then be identified (in the sense of the universality) with the temperature, or $t=T-T_{c}$, and the magnetic field, $h$, in the Ising model. One can easily understand this by realizing that the $t$ like direction should be tangential to the first-order line at the point E. Then $C_{V}$ and $-\partial^{2} \Omega / \partial \mu^{2}$ are different linear combinations of the $t$-like and $h$-like susceptibilities. In both linear combinations, the divergence of the $h$-like susceptibility will dominate because $\gamma \approx 1.2 \gg \alpha \approx 0.12$. The exponent for the divergence of the $h$-like susceptibility as a function of the distance, $\ell$, from the point $E$ will depend on the direction along which one approaches this point. For almost all directions it will be given by $\gamma / \beta \delta \approx 0.8$ (except for exactly the $t$-like direction, where it is $\gamma)$. As a result, for points on the $T \mu$ plane along a generic line through $\mathrm{E}$ one finds

$$
(\Delta T)^{2} \sim(\Delta \mu)^{2} \sim \ell^{0.8}
$$

sufficiently close to E. Therefore, the fluctuations of $T$ and $\mu$ are considerably suppressed when the freeze-out occurs near the critical point.

We turn now to direct signatures of the longwavelength fluctuations of the massless $\sigma$ field. For the choices of control parameters $x$ such that freeze-out occurs at (or near) the point $\mathrm{E}$, the $\sigma$-meson is the most numerous species at freeze-out, because it is (nearly) massless and so the equilibrium occupation number of the long-wavelength modes $(T / \omega)$ is large. Because the pions are massive at the critical point $\mathrm{E}$, the $\sigma$ 's cannot immediately decay into $\pi \pi$. Instead, they persist as the density of the system further decreases. It is important to realize that after freeze-out, one can (by definition) approximately neglect collisions between particles. Collective effects related to forward scattering amplitudes cannot be neglected. That is, although the particles no longer scatter, their dispersion relations will not be given by those in vacuum until the density is further reduced by continued expansion.

During the expansion, the in-medium sigma mass rises towards its vacuum value and eventually exceeds the $\pi \pi$ threshold. As the $\sigma \pi \pi$ coupling is large, the decay proceeds rapidly. This yields a population of pions with small transverse momentum, $p_{T}<m_{\pi}$. Because this process occurs after freeze-out, the pions generated by it 
do not get a chance to thermalize. Thus, the resulting pion spectrum should have a non-thermal enhancement at low $p_{T}$ which is largest for freeze-out near $\mathrm{E}$ where the $\sigma$ 's are most numerous.

We now propose a specific signature of the endpoint visible in the fluctuations of the pions resulting from the (formerly) long wavelength modes of the $\sigma$ field. For freeze-out close enough to $\mathrm{E}$ that the sigma mass at freeze-out is less than $T$, the thermal fluctuations of the number, $N_{\sigma}$, of $\sigma$ particles are determined by the classical statistics of the field $\sigma$, rather than by Poisson statistics of particles. Therefore, $\left\langle N_{\sigma}^{2}\right\rangle-\left\langle N_{\sigma}\right\rangle^{2} \sim\left\langle N_{\sigma}\right\rangle^{2}$, rather than $\left\langle N_{\sigma}\right\rangle$. Thus, we expect large event-by-event fluctuations in the multiplicity and distributions of the soft pions: $N_{\pi} \approx 2 N_{\sigma}$. Due to critical slowing down, non-equilibrium effects may further enhance these fluctuations. Thus, these pions could be detected either directly as an excess in the $p_{T}$-spectra at low $p_{T}$, or via increased event-by-event fluctuations at low $p_{T}$, or by an increase in Hanbury-Brown-Twiss (HBT) correlations due to the larger number of pions per phase space cell at low $p_{T}$. 27]

To conclude, we propose that by varying control parameters such as the collision energy and centrality, one may find a window of parameters for which the $T \mu$ trajectories pass close to the critical point E. Enhanced critical fluctuations of the $\sigma$ field and the associated thermodynamic singularities lead directly to the signatures we propose. When the freeze-out occurs near the point $\mathrm{E}$, we predict large non-thermal multiplicity and enhanced event-by-event fluctuations of the soft pions. In contrast, the event-by-event fluctuations in both $T$ and $\mu$, as determined using pions with $p_{T} \gtrsim m_{\pi}$, will be anomalously suppressed. Both effects should disappear if the atomic weight $A$ is very large. No one of these signatures is distinctive in isolation and without varying control parameters. Several of them seen together and seen to turn on and then turn off again as a control parameter is varied monotonously would constitute a decisive detection of the critical point.

What would we learn about QCD if such a point is found? First, we would learn that there is a genuine critical point in the $T \mu$ plane in nature. Second, we would learn that $m_{s}>m_{s 3}$ in nature, and the $\mu=0$ thermal transition is a crossover for physical quark masses, rather than a first-order phase transition. Third, the experimental discovery of the critical end-point $\mathrm{E}$ would mean that if the light quark masses were set to zero, there would be a tricritical point $\mathrm{P}$ in the phase diagram of QCD.

We are grateful to J. Berges, M. Halasz, A. Jackson, R. Shrock and J. Verbaarschot for fruitful collaborations inspiring this paper and helpful conversations. We also thank L. McLerran, G. Roland and M. Tsypin for helpful conversations. The work of MS is supported by NSF grant PHY97-22101. The work of KR is supported in part by the A.P. Sloan Foundation and by the DOE un- der agreement DE-FC02-94ER40818. The work of ES is supported in part by DOE grant DE-FG02-88ER40388.

[1] R. Pisarski and F. Wilczek, Phys. Rev. D29 (1984) 338.

[2] K. Rajagopal, Quark-Gluon Plasma 2, (World Scientific, 1995) 484, ed. R. Hwa.

[3] S.P. Klevansky, Rev. Mod. Phys. 64 (1992) 649; A. Barducci, R. Casalbuoni, G. Pettini and R. Gatto, Phys. Rev. D49 (1994) 426.

[4] M.A. Stephanov, Phys. Rev. Lett. 76 (1996) 4472; Nucl. Phys. B (Proc. Suppl.) 53 (1997) 469.

[5] M. Alford, K. Rajagopal and F. Wilczek, Phys. Lett. B422 (1998) 247 and hep-ph/9804403; R. Rapp, T. Schäfer, E. V. Shuryak and M. Velkovsky, hep$\mathrm{ph} / 9711396$.

[6] J. Berges and K. Rajagopal, hep-ph/9804233.

[7] M.A. Halasz, A.D. Jackson, R.E. Shrock, M.A. Stephanov and J.J.M. Verbaarschot, hep-ph/9804290.

[8] For a review, see I. Lawrie and S. Sarbach in Phase Transitions and Critical Phenomena 9, (Academic Press, 1984) 1, ed. C. Domb and J. Lebowitz.

[9] D. Bailin and A. Love, Phys. Rept. 107 (1984) 325.

[10] F. Wilczek, Int. J. Mod. Phys. A7 (1992) 3911; K. Rajagopal and F. Wilczek, Nucl. Phys. B399 (1993) 395.

[11] S. Gavin, A. Gocksch and R. Pisarski, Phys. Rev. D49 (1994) 3079.

[12] S. Hsu and M. Schwetz, hep-ph/9803386, version 3.

[13] Columbia Group, F.R. Brown et al, Phys. Rev. Lett. 65 (1990) 2491.

[14] See, e.g., P. Braun-Munziger and J. Stachel, Nucl. Phys. A606 (1996) 320.

[15] P. R. Subramanian, H. Stöcker and W. Greiner, Phys. Lett. B173 (1986) 468.

[16] C. M. Hung and E. Shuryak, Phys.Rev. C57 (1998) 1891.

[17] C. M. Hung and E. V. Shuryak, Phys. Rev. Lett. 75 (1995) 4003.

[18] D.H. Rischke, Y. Pursun, J. A. Maruhn, H. Stocker, W. Greiner, nucl-th/9505014.

[19] E. Shuryak and D. Teaney, Phys. Lett. B430 (1998) 37.

[20] L.P. Csernai and J.I. Kapusta, Phys. Rep. 131 (1986) 223.

[21] W. Trautmann, nucl-ex/9611002

[22] H.W. Barz, J.P. Bondorf, J.J. Gaardhoje, and H. Heiselberg, Phys. Rev. C57 (1998) 2536.

[23] U. Heinz, Proc. of Quark Matter '97, nucl-th/9801050.

[24] G. Roland for the NA49 collaboration, Proc. of Quark Matter '97 and Proc. of the Hirschegg Workshop on QCD Phase Transitions, 1997.

[25] L. Stodolsky, Phys. Rev. Lett. 75 (1995) 1044; E. V. Shuryak, Phys. Lett. B423 (1998) 9.

[26] See, e.g., K. Rummukainen, M. Tsypin, K. Kajantie, M. Laine, and M. Shaposhnikov, hep-lat/9805013.

[27] Note that this HBT signal is distinct from that proposed in E. Shuryak, Comments Nucl. Part. Phys. 21 (1994) 235 and J. Kapusta, D. Kharzeev and L. McLerran, Phys. 
Rev. D53 (1996) 5028. 